\title{
FETI-DP, BDDC, and Block Cholesky Methods
}

\author{
Jing $\mathrm{Li}^{*}$ and Olof Widlund ${ }^{\dagger}$
}

December 11, 2004

\begin{abstract}
Two popular non-overlapping domain decomposition methods, the FETI-DP and BDDC algorithms, are reformulated using Block Cholesky factorizations, an approach which can provide a useful framework for the design of domain decomposition algorithms for solving symmetric positive definite linear system of equations. Instead of introducing Lagrange multipliers to enforce the coarse level, primal continuity constraints in these algorithms, a change of variables is used such that each primal constraint corresponds to an explicit degree of freedom. With the new formulations of these algorithms, a simplified proof is provided that the spectra of a pair of FETI-DP and BDDC algorithms, with the same set of primal constraints, are the same. Results of numerical experiments also confirm this result.

Keywords: domain decomposition, FETI, Neumann-Neumann, BDDC, block Cholesky, primal constraints.

AMS subject classification: 65F05, 65F10, 65N55.
\end{abstract}

\section{Introduction}

The purpose of this paper is to give a simple derivation of two important domain decomposition methods namely the FETI-DP (dual-primal finite element tearing and interconnection) and the BDDC (balancing domain decomposition by constraints) algorithms. The latter, due to Clark Dohrmann,

\footnotetext{
${ }^{*}$ Department of Mathematical Sciences, Kent State University, Kent, OH 44242 li@math.kent.edu, URL: http://www.math.kent.edu/ li/

${ }^{\dagger}$ Courant Institute of Mathematical Sciences, New York University, 251 Mercer Street, New York, NY 10012 widlund@cs.nyu.edu, URL: http://www.cs.nyu.edu/cs/faculty/widlund/ This work was supported in part by the US Department of Energy under Contracts DE-FG02-92ER25127 and DE-FC02-01ER25482.
} 
$[13,2,1]$, represents an interesting redesign of the balancing NeumannNeumann algorithms with the coarse, global component of a BDDC algorithm expressed in terms of a set of primal constraints, just as in the FETIDP algorithms $[4,5]$. Throughout this paper, we will employ the language of block Cholesky elimination and our discussion can therefore also be seen as a guide to the design of domain decomposition methods using such a framework. We believe the success of this approach has been demonstrated by several of our friends who have quickly implemented FETI-DP and BDDC algorithms on the basis of an early version of this paper.

This paper is organized as follows. We first consider the case of two subdomains and two dimensions. We show that the iteration matrices of the standard Neumann-Neumann and one-level FETI methods are very closely related and that they have the same eigenvalues. A FETI-DP algorithm is then introduced in terms of a single primal constraint, which enforces the continuity of an edge average. A minimum requirement is then met that the quadratic form defined by the sum of the quadratic forms, formed with the stiffness matrices of the subdomains and subject to the primal constraints, is positive definite. In any such a case, the subdomain problems are positive definite if homogeneous boundary conditions corresponding to the primal constraints of the subdomain are imposed. We note that in contrast to the older balancing Neumann-Neumann and one-level FETI methods, the FETI-DP and BDDC methods do not require the solution of any linear systems of equations with singular matrices.

We then derive, for the case of many subdomains, a Schur complement matrix $\widetilde{\mathcal{S}}_{\Gamma}$ which represents a partially assembled system matrix obtained from the stiffness matrices of the individual subdomains. This Schur complement is constructed by eliminating, in parallel across the subdomains, the sets of interior degrees of freedom and then assembling the system by making the primal variables, and the primal variables only, global. The primal variables, which will have the same values across the interface throughout the iteration, will be a selection of nodal degrees of freedom and, quite importantly, certain averages and first order moments over edges and faces of the interface formed by the parts of the boundaries of the subdomains that are common to more than one subdomain.

We show that all main building blocks of the FETI-DP and BDDC algorithms can be expressed in terms of $\widetilde{\mathcal{S}}_{\Gamma}$ and its inverse and certain simple restriction, extension, and scaling matrices. We then provide a new, short proof of an important result, due to Mandel, Dohrmann, and Tezaur [14], which shows that the eigenvalues of a pair of FETI-DP and BDDC algorithms, with the same sets of primal constraints, are the same. We note that 
this fact was first observed experimentally by Fragakis and Papadrakakis [6]; these authors also discussed primal iterative substructuring methods which are close counterparts to FETI algorithms. A consequence of the result in $[6,14]$ is that there is no real difference between the convergence rates of a FETI-DP and a BDDC algorithm with the same set of primal constraints. The choice of algorithm can therefore be based on other considerations; we note, in particular, that it appears easier to modify BDDC than FETI-DP when introducing additional levels; cf. Tu [17]. Finally, we provide some numerical results which indeed support the main result on the spectra of the operators.

We note that the choice of the primal constraints is a core question for any efficient FETI-DP or BDDC algorithm. This issue will not be addressed in this paper; see instead Klawonn, Widlund and Dryja [10, 11], Klawonn and Widlund [9], and Klawonn and Rheinbach [7]. It has been established, in several cases, that the condition number of the preconditioned operator is bounded by $C(1+\log (H / h))^{2}$ if the primal constraints and certain diagonal scalings are well chosen; see, e.g., $[15,10,9]$. Here, $C$ is a constant independent of the number of subdomains as well as the size of the elements, and $H / h$ is the maximum number of elements across any subdomain. In this paper, we will only assume that some choice of primal constraints has been made and that it satisfies the minimal requirement of positive definiteness.

To simplify the notation and analysis, we will make a change of the variables so that, e.g., a primal constraint related to the average of the solution over an edge, or the average of a component of the displacement, which should have a common value irrespective of which component of the product space is used in its computation, will correspond to one of the new primal variables. This change of variables, which will be discussed in some detail in an illustrative case in Section 3.3, will also result in a new set of dual displacement variables representing the degrees of freedom for which the primal constraints vanish; they span the dual displacement space. The change of variables separates the primal and dual variables and make our arguments much easier; this approach can also be the basis for computational practice as in Klawonn and Widlund [9, Section 6.2] and Klawonn and Rheinbach [7].

For further references to domain decomposition methods of FETI and balancing type, see Toselli and Widlund [16], in particular Section 6.1, and the introductory parts of Sections 6.2, 6.3, and 6.4. 


\section{Block Cholesky Elimination}

Let us first consider how to represent the inverse of a symmetric, positive definite block matrix

$$
\left[\begin{array}{cc}
A & B^{T} \\
B & C
\end{array}\right]
$$

Using block Cholesky elimination, we have

$$
\left[\begin{array}{cc}
A & B^{T} \\
B & C
\end{array}\right]=\left[\begin{array}{cc}
I_{A} & \\
B A^{-1} & I_{C}
\end{array}\right]\left[\begin{array}{cc}
A & \\
& C-B A^{-1} B^{T}
\end{array}\right]\left[\begin{array}{cc}
I_{A} & A^{-1} B^{T} \\
& I_{C}
\end{array}\right]
$$

where $I_{A}$ and $I_{C}$ are appropriate identity matrices. The matrix $\mathcal{S}=C-$ $B A^{-1} B^{T}$ is a Schur complement, which is always positive definite. Inverting the three factors, we find that

$$
\begin{aligned}
{\left[\begin{array}{cc}
A & B^{T} \\
B & C
\end{array}\right]^{-1} } & =\left[\begin{array}{cc}
I_{A} & -A^{-1} B^{T} \\
& I_{C}
\end{array}\right]\left[\begin{array}{ll}
A^{-1} & \\
& \mathcal{S}^{-1}
\end{array}\right]\left[\begin{array}{cc}
I_{A} & \\
-B A^{-1} & I_{C}
\end{array}\right](2) \\
& =\left[\begin{array}{cc}
A^{-1} & 0 \\
0 & 0
\end{array}\right]+\Phi \mathcal{S}^{-1} \Phi^{T}
\end{aligned}
$$

where

$$
\Phi=\left[\begin{array}{c}
-A^{-1} B^{T} \\
I_{C}
\end{array}\right]
$$

It is easy to see that applying this inverse to a vector will involve solving two linear systems with the matrix $A$ and one linear system with the matrix $\mathcal{S}$.

We can think of $\Phi$ as the extension of the columns of the identity block matrix $I_{C}$ to the other, the $A$-block, part. We can also regard the use of $\Phi$ as a change of variables which reduces the operator to block diagonal form. We then use a basis defined by the columns of the first factor, of the three in Equation (2).

In our applications, the matrix $A$ will be a direct sum of many submatrices corresponding to the subdomains into which a given domain has been partitioned while the second diagonal block, $C$, will represent the global part of a preconditioner representing a select few of the degrees of freedom of the interface between the subdomains. We note that all the interface variables must belong to the second set of variables if we are developing an exact solver. However, powerful preconditioners can be developed using remarkably few global, primal degrees of freedom associated with the matrix $C$; in such a case the remaining interface degrees of freedom are made part of the 
local, dual part of the space of interface displacements and of the matrix $A$. We can also exploit ways of computing $\mathcal{S}$ by assembling contributions from the local problems. It is clear that we should be anxious to keep the order of $C$ and $\mathcal{S}$ small since factoring $\mathcal{S}$ can be quite expensive and does not parallelize as well as the rest.

\section{The Two Subdomain Case}

We begin by considering the two subdomain case and a domain $\Omega$ subdivided into two nonoverlapping subdomains $\Omega_{1}$ and $\Omega_{2}$. The interface between these two open set is $\Gamma=\left(\partial \Omega_{1} \cup \partial \Omega_{2}\right) \backslash \partial \Omega$, as shown in Figure 1 ; we could equally well consider a problem in three dimensions.

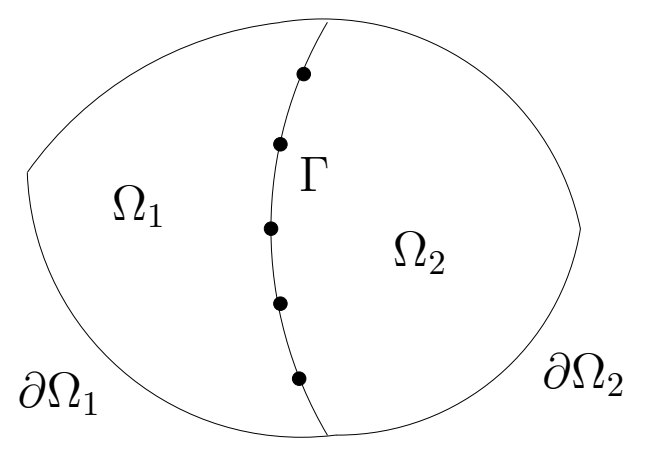

Figure 1: Partition into two nonoverlapping subdomains.

We introduce triangulations of the $\Omega_{i}$, with common nodal points on the interface, and a finite element approximation of a second order, selfadjoint, elliptic problem such as a scalar elliptic problem or the equations of linear elasticity. We also provide standard boundary values and volume data; for simplicity, we first assume that we have a zero Dirichlet condition on all of $\partial \Omega$ and that the boundaries of both subdomains intersect that set.

We begin by computing a stiffness matrix and a load vector for each subdomain

$$
A^{(i)}=\left[\begin{array}{cc}
A_{I I}^{(i)} & A_{I \Gamma}^{(i)} \\
A_{\Gamma I}^{(i)} & A_{\Gamma \Gamma}^{(i)}
\end{array}\right], \quad f^{(i)}=\left[\begin{array}{c}
f_{I}^{(i)} \\
f_{\Gamma}^{(i)}
\end{array}\right], \quad u^{(i)}=\left[\begin{array}{c}
u_{I}^{(i)} \\
u_{\Gamma}^{(i)}
\end{array}\right], \quad i=1,2 .
$$

This provides a finite element model for each of the subdomains with the 
given Dirichlet boundary data on $\partial \Omega_{i} \backslash \Gamma$ and a homogeneous Neumann condition on $\Gamma$.

The finite element model for the entire problem is obtained by assembling the two subproblems:

$$
A=\left[\begin{array}{ccc}
A_{I I}^{(1)} & 0 & A_{I \Gamma}^{(1)} \\
0 & A_{I I}^{(2)} & A_{I \Gamma}^{(2)} \\
A_{\Gamma I}^{(1)} & A_{\Gamma I}^{(2)} & A_{\Gamma \Gamma}
\end{array}\right], \quad f=\left[\begin{array}{c}
f_{I}^{(1)} \\
f_{I}^{(2)} \\
f_{\Gamma}
\end{array}\right], \quad u=\left[\begin{array}{c}
u_{I}^{(1)} \\
u_{I}^{(2)} \\
u_{\Gamma}
\end{array}\right]
$$

Here, $A_{\Gamma \Gamma}=A_{\Gamma \Gamma}^{(1)}+A_{\Gamma \Gamma}^{(2)}$ and $f_{\Gamma}=f_{\Gamma}^{(1)}+f_{\Gamma}^{(2)}$ and they represent sums of contributions from the individual subdomains. The degrees of freedom are partitioned into those interior to $\Omega_{1}, \Omega_{2}$, and those on $\Gamma$, respectively.

After eliminating the interior unknowns of each subdomain separately, we obtain two subdomain Schur complement operators and two subdomain interface load vectors: for $i=1,2$,

$$
\begin{aligned}
\mathcal{S}^{(i)} & :=A_{\Gamma \Gamma}^{(i)}-A_{\Gamma I}^{(i)} A_{I I}^{(i)}{ }^{-1} A_{I \Gamma}^{(i)}, \\
g_{\Gamma}^{(i)} & :=f_{\Gamma}^{(i)}-A_{\Gamma I}^{(i)} A_{I I}^{(i)} f_{I}^{(i)} .
\end{aligned}
$$

The given system, $A u=f$, is reduced to

$$
\left(\mathcal{S}^{(1)}+\mathcal{S}^{(2)}\right) u_{\Gamma}=g_{\Gamma}^{(1)}+g_{\Gamma}^{(2)} .
$$

We note that we can compute $\mathcal{S}^{(i)}$ times a vector by a local computation involving $\Omega_{i}$ only; the main effort is the solution of a Dirichlet problem and there are also some sparse matrix-vector products. Similarly, we can find $\mathcal{S}^{(i)^{-1}} g_{\Gamma}^{(i)}$ by solving a linear system with the matrix $A^{(i)}$ and with a right hand side $\left(0, g_{\Gamma}^{(i)}\right)^{T}$.

\subsection{A Neumann-Neumann Method}

In this simple case, the basic Neumann-Neumann preconditioner,

$$
M^{-1}=\mathcal{S}^{(1)^{-1}}+\mathcal{S}^{(2)^{-1}}
$$

can be used for solving the interface problem (8). Thus, instead of working with the inverse of the sum of the two Schur complements, we use the sum of the inverses. All solves are then for problems on subdomains, since, as already pointed out, the action of $\mathcal{S}^{(i)^{-1}}$ on a vector $g_{\Gamma}^{(i)}$ can be obtained by 
solving a local linear system. The rate of convergence of the method depends on the eigenvalues of the generalized eigenvalue problem $\mathcal{S}^{(1)} \phi=\lambda \mathcal{S}^{(2)} \phi$ since the preconditioned operator, $\left(\mathcal{S}^{(1)^{-1}}+\mathcal{S}^{(2)^{-1}}\right)\left(\mathcal{S}^{(1)}+\mathcal{S}^{(2)}\right)$, equals $2 I+\mathcal{S}^{(1)^{-1}} \mathcal{S}^{(2)}+\mathcal{S}^{(2)^{-1}} \mathcal{S}^{(1)}$. It can be established that the eigenvalues of the generalized eigenvalue problem are uniformly bounded from above as well as uniformly bounded away from zero; see [16, Section 1.3].

\section{$3.2 \quad$ A FETI Method}

We now consider two local mixed Neumann-Dirichlet problems:

$$
\left[\begin{array}{ll}
A_{I I}^{(i)} & A_{I \Gamma}^{(i)} \\
A_{\Gamma I}^{(i)} & A_{\Gamma \Gamma}^{(i)}
\end{array}\right]\left[\begin{array}{c}
u_{I}^{(i)} \\
u_{\Gamma}^{(i)}
\end{array}\right]=\left[\begin{array}{c}
f_{I}^{(i)} \\
f_{\Gamma}^{(i)}+\lambda_{\Gamma}^{(i)}
\end{array}\right], \quad i=1,2 .
$$

Here, $\lambda_{\Gamma}=\lambda_{\Gamma}^{(1)}=-\lambda_{\Gamma}^{(2)}$ is the unknown flux; once it has been found, we can obtain the solution by solving the two local problems; see [16, Section 1.3.5] for more details. We obtain, with $g_{\Gamma}^{(i)}=f_{\Gamma}^{(i)}-A_{\Gamma I}^{(i)} A_{I I}^{(i)}{ }^{-1} f_{I}^{(i)}$,

$$
u_{\Gamma}^{(i)}=\mathcal{S}^{(i)^{-1}}\left(g_{\Gamma}^{(i)}+\lambda_{\Gamma}^{(i)}\right) .
$$

Using the requirement that $u_{\Gamma}^{(1)}=u_{\Gamma}^{(2)}$, we obtain $F \lambda_{\Gamma}=d_{\Gamma}$, with $F=$ $\mathcal{S}^{(1)^{-1}}+\mathcal{S}^{(2)^{-1}}$. We precondition this interface equation with $\mathcal{S}^{(1)}+\mathcal{S}^{(2)}$, and we find that, $\left(\mathcal{S}^{(1)}+\mathcal{S}^{(2)}\right)\left(\mathcal{S}^{(1)^{-1}}+\mathcal{S}^{(2)^{-1}}\right)$, the resulting preconditioned operator, has the same eigenvalues as the preconditioned operator of Section 3.1.

We note that we can reformulate the problem as a constrained minimization problem, minimizing the sum of the energy forms of the two subproblems subject to the constraint $u_{\Gamma}^{(1)}-u_{\Gamma}^{(2)}=0$. We then see that $\lambda_{\Gamma}$ will be a vector of Lagrange multipliers; see further the discussion in Section 3.3.

\subsection{A FETI-DP Method and a Change of Variables}

The FETI-DP methods were first introduced for two-dimensional problems by Farhat, Lesoinne, LeTallec, Pierson, and Rixen [4]. In their algorithms, continuity of the primal variables at the subdomain vertices is maintained (by subassembly), while other continuity constraints are enforced by Lagrange multipliers but only fully so at the convergence of the algorithm. In this section, we give an example of a two-dimensional, two-subdomain case, where there is a Dirichlet boundary condition on part of $\partial \Omega_{1}$ and a 
Neumann boundary condition elsewhere as shown in Figure 2. Instead of a vertex constraint, we select the interface average as the sole primal variable. We specialize to a scalar elliptic equation, which has a one-dimensional null space of constants in the case of Neumann boundary conditions. We note that we equally well could have treated a three-dimensional problem with a constraint expressed in terms of a face average or an average over an edge of the face, which is common to the two subdomains. For a three-dimensional elasticity problem, six constraints would be needed on the interface to prevent the subdomain $\Omega_{2}$ from floating.

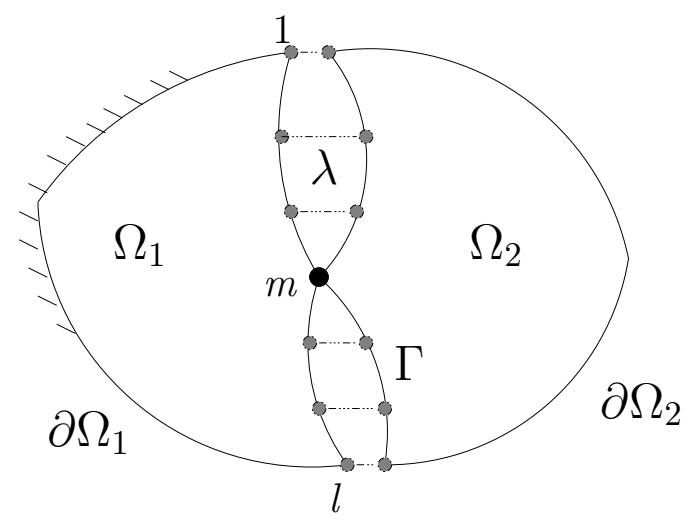

Figure 2: Partition into two subdomains, with $\Omega_{2}$ floating, in the absence of a constraint.

We will first show how we can change variables to make the edge average degree of freedom explicit. Denote the unknowns corresponding to the nodal degrees of freedom on the interface edge $\Gamma$ by $\left(u_{1}, \ldots, u_{m}, \ldots, u_{l}\right)$, where the node $m$ can be any node on the edge. A linear system for a subdomain can be written as

$$
A^{(i)} u^{(i)}:=\left[\begin{array}{cccccc}
A_{I I}^{(i)} & A_{1 I}^{(i)^{T}} & \ldots & A_{m I}^{(i)^{T}} & \ldots & A_{l I}^{(i)^{T}} \\
A_{1 I}^{(i)} & a_{11}^{(i)} & \ldots & a_{1 m}^{(i)} & \ldots & a_{1 l}^{(i)} \\
\vdots & \vdots & \ddots & \vdots & & \vdots \\
A_{m I}^{(i)} & a_{m 1}^{(i)} & \ldots & a_{m m}^{(i)} & \ldots & a_{m l}^{(i)} \\
\vdots & \vdots & & \vdots & \ddots & \vdots \\
A_{l I}^{(i)} & a_{l 1}^{(i)} & \ldots & a_{l m}^{(i)} & \ldots & a_{l l}^{(i)}
\end{array}\right]\left[\begin{array}{c}
u_{I}^{(i)} \\
u_{1}^{(i)} \\
\vdots \\
u_{m}^{(i)} \\
\vdots \\
u_{l}^{(i)}
\end{array}\right]=\left[\begin{array}{c}
f_{I}^{(i)} \\
f_{1}^{(i)} \\
\vdots \\
f_{m}^{(i)} \\
\vdots \\
f_{l}^{(i)}
\end{array}\right]
$$

Nodal values on $\partial \Omega$, where Neumann conditions are imposed, are part of the 
vectors of interior values $u_{I}^{(i)}$. The interface variables of both subdomains can be changed to

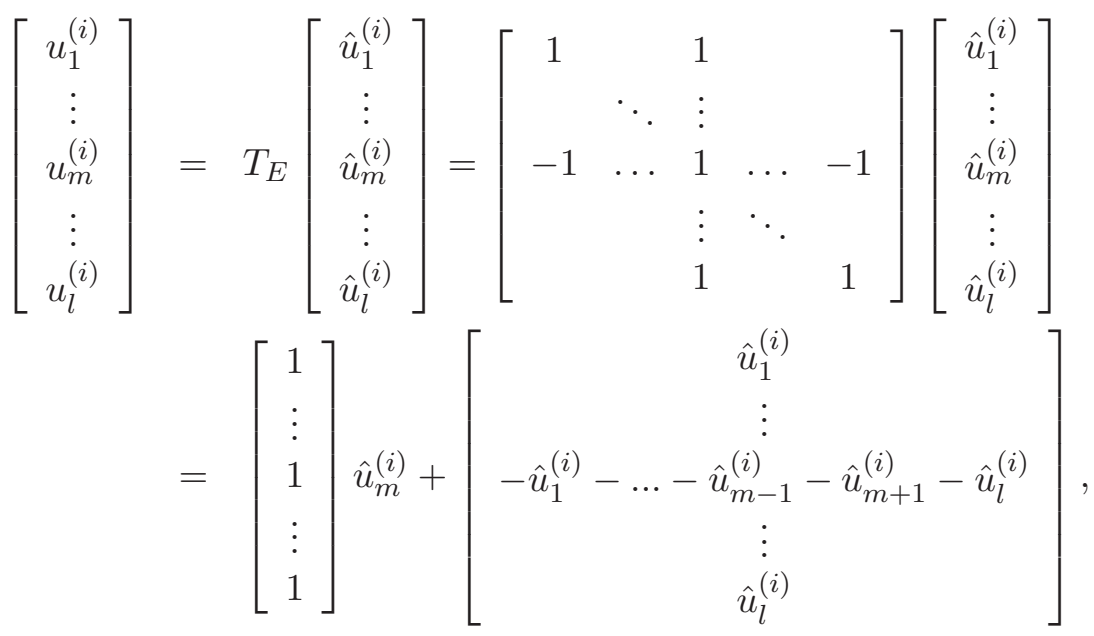

where $T_{E}$ is the $l$ by $l$ square matrix shown above, with columns representing the new basis of the space of edge variables. The original interface nodal unknowns have now been separated into two parts. The first part corresponds to a basis function, which is constant on the edge and has a value $\hat{u}_{m}^{(i)}$ for the subdomain $\Omega_{i}$. The second corresponds to functions with zero edge averages. Correspondingly, the transformed subdomain problem is of the form

$$
T^{T}\left[\begin{array}{cccccc}
A_{I I}^{(i)} & A_{1 I}^{(i)^{T}} & \ldots & A_{m I}^{(i)^{T}} & \ldots & A_{l I}^{(i)^{T}} \\
A_{1 I}^{(i)} & a_{11}^{(i)} & \ldots & a_{1 m}^{(i)} & \ldots & a_{1 l}^{(i)} \\
\vdots & \vdots & \ddots & \vdots & & \vdots \\
A_{m I}^{(i)} & a_{m 1}^{(i)} & \ldots & a_{m m}^{(i)} & \ldots & a_{m l}^{(i)} \\
\vdots & \vdots & & \vdots & \ddots & \vdots \\
A_{l I}^{(i)} & a_{l 1}^{(i)} & \ldots & a_{l m}^{(i)} & \ldots & a_{l l}^{(i)}
\end{array}\right] T\left[\begin{array}{c}
u_{I}^{(i)} \\
\hat{u}_{1}^{(i)} \\
\vdots \\
\hat{u}_{m}^{(i)} \\
\vdots \\
\hat{u}_{l}^{(i)}
\end{array}\right]=T^{T}\left[\begin{array}{c}
f_{I}^{(i)} \\
f_{1}^{(i)} \\
\vdots \\
f_{m}^{(i)} \\
\vdots \\
f_{l}^{(i)}
\end{array}\right]
$$

where $T$ is a diagonal block matrix of the form

$$
T=\left[\begin{array}{ll}
I & \\
& T_{E}
\end{array}\right]
$$

We note that the change of basis on an individual interface edge is a local procedure, i.e., it can be carried out edge by edge, as long as the sets of variables being transformed do not contain any common degrees of freedom. 
We will, from now on, always assume that the subdomain variables have been changed, when primal edges or faces are being used. In other words, we will have explicit primal unknowns for the subdomain problem, which correspond to edge or face average finite element basis functions.

In the example shown in Figure 2, the subdomain edge average degrees of freedom, $\hat{u}_{m}^{(1)}$ and $\hat{u}_{m}^{(2)}$, are required to have a common value throughout the FETI-DP iteration. From now on, we will denote this common degree of freedom by $u_{\Pi}$, a notation often used for the vector of global, coarselevel, primal variables. The other interface degrees of freedom are $u_{\Delta}^{(1)}$ and $u_{\Delta}^{(2)}$, the dual interface variables, for the neighboring subdomains with their own values at the same interface nodes. The global matrix problem of our example can then be written as

$$
\left[\begin{array}{cccccc}
A_{I I}^{(1)} & A_{\Delta I}^{(1)^{T}} & & & A_{\Pi I}^{(1)^{T}} & \\
A_{\Delta I}^{(1)} & A_{\Delta \Delta}^{(1)} & & & A_{\Pi \Delta}^{(1)^{T}} & B_{\Delta}^{(1)^{T}} \\
& & A_{I I}^{(2)} & A_{\Delta I}^{(2)^{T}} & A_{\Pi I}^{(2)^{T}} & \\
& & A_{\Delta I}^{(2)} & A_{\Delta \Delta}^{(2)} & A_{\Pi \Delta}^{(2)^{T}} & B_{\Delta}^{(2)^{T}} \\
A_{\Pi I}^{(1)} & A_{\Pi \Delta}^{(1)} & A_{\Pi I}^{(2)} & A_{\Pi \Delta}^{(2)} & A_{\Pi \Pi}^{(1)}+A_{\Pi \Pi}^{(2)} & \\
& B_{\Delta}^{(1)} & & B_{\Delta}^{(2)} & &
\end{array}\right]\left[\begin{array}{c}
u_{I}^{(1)} \\
u_{\Delta}^{(1)} \\
u_{I}^{(2)} \\
u_{\Delta}^{(2)} \\
u_{\Pi} \\
\lambda
\end{array}\right]=\left[\begin{array}{c}
f_{I}^{(1)} \\
f_{\Delta}^{(1)} \\
f_{I}^{(2)} \\
f_{\Delta}^{(2)} \\
f_{\Pi}^{(1)}+f_{\Pi}^{(2)} \\
0
\end{array}\right] .
$$

Here the matrices $B_{\Delta}^{(i)}$ have elements from the set $\{0,1,-1\}$ and are chosen such that the $l-1$ equations of $B_{\Delta}^{(1)} u_{\Delta}^{(1)}+B_{\Delta}^{(2)} u_{\Delta}^{(2)}=0$, are $u_{k}^{(1)}-u_{k}^{(2)}=0$, for $k=1, \ldots, m-1, m+1, \ldots, l$, which guarantees continuity of the dual variables $u_{\Delta}^{(1)}$ and $u_{\Delta}^{(2)}$ across the interface; $\lambda$ is the corresponding vector of Lagrange multipliers and has a length of $l-1$ in this example.

We now eliminate the local variables $u_{I}^{(1)}, u_{\Delta}^{(1)}, u_{I}^{(2)}$, and $u_{\Delta}^{(2)}$, and we obtain:

$$
\left[\begin{array}{cc}
\mathcal{S}_{\Pi \Pi} & \widetilde{B}_{\Lambda \Pi}^{T} \\
\widetilde{B}_{\Lambda \Pi} & \widetilde{B}_{\Lambda \Lambda}
\end{array}\right]\left[\begin{array}{c}
u_{\Pi} \\
\lambda
\end{array}\right]=\left[\begin{array}{l}
g_{\Pi} \\
d_{\Lambda}
\end{array}\right]
$$

where,

$$
\mathcal{S}_{\Pi \Pi}=\sum_{i=1}^{2} R_{\Pi}^{(i)^{T}}\left(A_{\Pi \Pi}^{(i)}-\left[\begin{array}{ll}
A_{\Pi I}^{(i)} & A_{\Pi \Delta}^{(i)}
\end{array}\right]\left[\begin{array}{cc}
A_{I I}^{(i)} & A_{\Delta I}^{(i)^{T}} \\
A_{\Delta I}^{(i)} & A_{\Delta \Delta}^{(i)}
\end{array}\right]^{-1}\left[\begin{array}{c}
A_{\Pi I}^{(i)^{T}} \\
A_{\Pi \Delta}^{(i)^{T}}
\end{array}\right]\right) R_{\Pi}^{(i)},
$$




$$
\begin{aligned}
\widetilde{B}_{\Lambda \Pi} & =-\sum_{i=1}^{2}\left[\begin{array}{ll}
0 & B_{\Delta}^{(i)}
\end{array}\right]\left[\begin{array}{cc}
A_{I I}^{(i)} & A_{\Delta I}^{(i)^{T}} \\
A_{\Delta I}^{(i)} & A_{\Delta \Delta}^{(i)}
\end{array}\right]^{-1}\left[\begin{array}{c}
A_{\Pi I}^{(i)^{T}} \\
A_{\Pi \Delta}^{(i)^{T}}
\end{array}\right] R_{\Pi}^{(i)}, \\
\widetilde{B}_{\Lambda \Lambda} & =-\sum_{i=1}^{2}\left[\begin{array}{ll}
0 & B_{\Delta}^{(i)}
\end{array}\right]\left[\begin{array}{cc}
A_{I I}^{(i)} & A_{\Delta I}^{(i)^{T}} \\
A_{\Delta I}^{(i)} & A_{\Delta \Delta}^{(i)}
\end{array}\right]^{-1}\left[\begin{array}{c}
0 \\
B_{\Delta}^{(i)^{T}}
\end{array}\right], \\
g_{\Pi} & =\sum_{i=1}^{2} R_{\Pi}^{(i)^{T}}\left(f_{\Pi}^{(i)}-\left[\begin{array}{ll}
A_{\Pi I}^{(i)} & A_{\Pi \Delta}^{(i)}
\end{array}\right]\left[\begin{array}{cc}
A_{I I}^{(i)} & A_{\Delta I}^{(i)^{T}} \\
A_{\Delta I}^{(i)} & A_{\Delta \Delta}^{(i)}
\end{array}\right]^{-1}\left[\begin{array}{c}
f_{I}^{(i)} \\
f_{\Delta}^{(i)}
\end{array}\right]\right), \\
d_{\Lambda} & =-\sum_{i=1}^{2}\left[\begin{array}{ll}
0 & B_{\Delta}^{(i)}
\end{array}\right]\left[\begin{array}{ll}
A_{I I}^{(i)} & A_{\Delta I}^{(i)^{T}} \\
A_{\Delta I}^{(i)} & A_{\Delta \Delta}^{(i)}
\end{array}\right]^{-1}\left[\begin{array}{c}
f_{I}^{(i)} \\
f_{\Delta}^{(i)}
\end{array}\right],
\end{aligned}
$$

and where $R_{\Pi}^{(i)}$ is the matrix, with $\{0,1\}$ elements, which maps the global primal variable $u_{\Pi}$ to its subdomain component $u_{\Pi}^{(i)}$. In this simple example, both $R_{\Pi}^{(1)}$ and $R_{\Pi}^{(2)}$ are equal to 1 .

We note that the second block in the leading block diagonal part of Equation (11) is invertible, since we have imposed the edge average constraint.

Equation (12) is further reduced to a linear system of equations for the Lagrange multiplier $\lambda$ :

$$
\left(\widetilde{B}_{\Lambda \Lambda}-\widetilde{B}_{\Lambda \Pi} \mathcal{S}_{\Pi \Pi}^{-1} \widetilde{B}_{\Lambda \Pi}^{T}\right) \lambda=d_{\Lambda}-\widetilde{B}_{\Lambda \Pi} \mathcal{S}_{\Pi \Pi}^{-1} g_{\Pi} .
$$

A Dirichlet preconditioner is often used in FETI-DP algorithms for solving Equation (18) and it will be discussed in Section 4.1. We also typically use a conjugate gradient method to accelerate the convergence.

We end this section with a few comments on a BDDC algorithm for this case. The basic step of a BDDC preconditioner involves the solution of a positive definite, symmetric linear system with a matrix obtained from that of (11) by dropping the rows and columns related to the constraints and the Lagrange mulitplier. Solving such a linear system will typically result in a vector such that $u_{\Delta}^{(1)} \neq u_{\Delta}^{(2)}$. Continuity is then restored by computing a weighted average of these vectors. Thereafter, the fully assembled residual of the resulting vector is computed, the residual is then mapped into the appropriate space of right hand sides of the partially assembled system by using the transpose of the average operator, and the steps are then repeated; see further Section 4.2. 


\section{Many Subdomains}

We will now divide the region $\Omega$ into many subdomains $\Omega_{i}$. The interface between the subdomains is defined by

$$
\Gamma:=\left(\cup_{i \neq j} \partial \Omega_{i} \cap \partial \Omega_{j}\right) \backslash \partial \Omega_{D},
$$

where $\partial \Omega_{D}$ is a nonempty subset of $\partial \Omega$ corresponding to the Dirichlet boundary conditions; the interface of the subdomain $\Omega_{i}$ is defined by $\Gamma_{i}=\partial \Omega_{i} \cap \Gamma$. We will denote a finite element space of continuous functions on $\Omega_{i}$ by $W^{(i)}$; we will always assume that these functions vanish on $\partial \Omega_{i} \cap \partial \Omega_{D}$. Each $W^{(i)}$ is decomposed into a subdomain interior part and a subdomain interface part,

$$
W^{(i)}=W_{I}^{(i)} \bigoplus W_{\Gamma}^{(i)}
$$

The associated product spaces are denoted by $W:=\prod_{i=1}^{N} W^{(i)}, W_{I}:=$ $\prod_{i=1}^{N} W_{I}^{(i)}$, and $W_{\Gamma}:=\prod_{i=1}^{N} W_{\Gamma}^{(i)}$, respectively.

The finite element solutions are continuous across the interface and we denote the corresponding subspace of $W_{\Gamma}$ by $\widehat{W}_{\Gamma}$; generally the functions in the space $W_{\Gamma}$ are discontinuous across the interface. We also introduce a subspace $\widetilde{W}_{\Gamma}$, intermediate between $\widehat{W}_{\Gamma}$ and $W_{\Gamma}$, which can be written as

$$
\widetilde{W}_{\Gamma}=W_{\Delta} \bigoplus \widehat{W}_{\Pi}=\left(\prod_{i=1}^{N} W_{\Delta}^{(i)}\right) \bigoplus \widehat{W}_{\Pi},
$$

where $\widehat{W}_{\Pi}$ is the continuous, coarse-level, primal variable space. $\widehat{W}_{\Pi}$ is typically spanned by subdomain corner nodal basis functions, and/or by interface edge and/or face basis functions with constant values at the nodes of the edge or face. $W_{\Delta}$ is the product space of subdomain dual interface variable spaces $W_{\Delta}^{(i)}$, which consists of functions with zero values at the primal degrees of freedom. Here, as always, we assume that the basis has been changed so that each primal constraint corresponds to an explicit degree of freedom.

Several restriction and extension operators between these interface spaces need to be defined. $R_{\Gamma \Delta}$ and $R_{\Gamma \Pi}$ are the restriction operators from the space $\widetilde{W}_{\Gamma}$ onto its subspaces $W_{\Delta}$ and $\widehat{W}_{\Pi} \cdot R_{\Gamma}^{(i)}: \widehat{W}_{\Gamma} \rightarrow W_{\Gamma}^{(i)}, R_{\Delta}^{(i)}: W_{\Delta} \rightarrow W_{\Delta}^{(i)}$, and $R_{\Pi}^{(i)}: \widehat{W}_{\Pi} \rightarrow W_{\Pi}^{(i)}$, correspondingly map global interface vectors to its component on $\Gamma_{i}$, respectively. $R_{\Gamma}: \widehat{W}_{\Gamma} \rightarrow W_{\Gamma}$, is the direct sum of the $R_{\Gamma}^{(i)} . \widetilde{R}_{\Gamma}: \widehat{W}_{\Gamma} \rightarrow \widetilde{W}_{\Gamma}$ is the direct sum of $R_{\Gamma \Pi}$ and the $R_{\Delta}^{(i)} R_{\Gamma \Delta}$. 
In order to define certain scaling operators, we need to introduce a positive scaling factor $\delta_{i}^{\dagger}(x)$ for any node $x$ on the interface $\Gamma_{i}$ of the subdomain $\Omega_{i}$. In applications, these scaling factors will depend on the heat conduction coefficient and the first of the Lamé parameters for scalar elliptic problems and the equations of linear elasticity, respectively; see [10,9]. Here, with $\mathcal{N}_{x}$ the set of indices of the subregions which has $x$ on its boundary, we will only assume that $\sum_{j \in \mathcal{N}_{x}} \delta_{j}^{\dagger}(x)=1$. Given the scaling factors at the subdomain interface nodes, we can define scaled operators $R_{D, \Gamma}^{(i)}$ and $R_{D, \Delta}^{(i)}$. We note that each row of $R_{\Gamma}^{(i)}$ and $R_{\Delta}^{(i)}$ has only one nonzero entry which corresponds to a subdomain interface node $x$. Multiplying each such row with the scaling factor $\delta_{i}^{\dagger}(x)$ gives us $R_{D, \Gamma}^{(i)}$ and $R_{D, \Delta}^{(i)}$, respectively. The scaled operators $R_{D, \Gamma}$ and $\widetilde{R}_{D, \Gamma}$ are direct sums of $R_{D, \Gamma}^{(i)}$, and of $R_{\Gamma \Pi}$ and $R_{D, \Delta}^{(i)} R_{\Gamma \Delta}$, respectively.

\subsection{FETI-DP Algorithms}

In a FETI-DP algorithm, the finite element space is decomposed as

$$
W=W_{I} \bigoplus \widetilde{W}_{\Gamma}=W_{I} \bigoplus W_{\Delta} \bigoplus \widehat{W}_{\Pi} .
$$

If we have primal vertex constraints only, the corresponding finite element model can be regarded as obtained after having made incisions which remove the coupling between the nodes on the subdomain interface, except at subdomain vertices as shown in Figure 3.

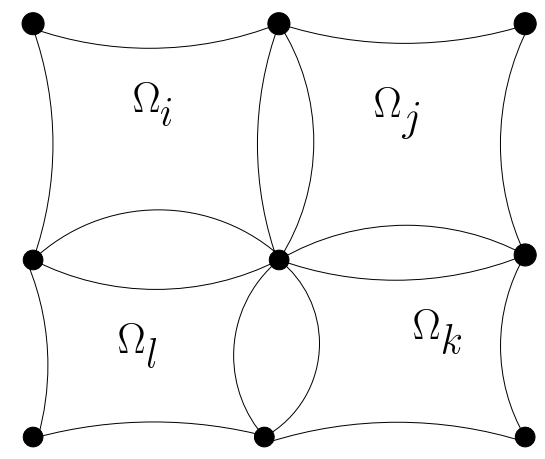

Figure 3: Decomposition of subdomains for a FETI-DP method.

The global linear system of equations is a simple generalization of Equation (11) to the many subdomain case, and the reduced equation for the 
Lagrange multiplier $\lambda$ is of the form (18) where the corresponding operators and vectors are defined as in Equations (13)-(17). The space of Lagrange multipliers is $V=B_{\Delta} W_{\Delta}$, where $B_{\Delta}$ is defined in terms of subdomain operators $B_{\Delta}^{(i)}$, and the continuity constraints across the interface are written as

$$
B_{\Delta} u_{\Delta}=\sum_{i=1}^{N} B_{\Delta}^{(i)} u_{\Delta}^{(i)}=0
$$

In the following, we will rewrite the FETI-DP operator of Equation (18) in terms of certain Schur complements, and also introduce a Dirichlet preconditioner.

We define a Schur complement operator $\widetilde{\mathcal{S}}_{\Gamma}$ on the space $\widetilde{W}_{\Gamma}$ by: given interface variables $w_{\Gamma} \in \widetilde{W}_{\Gamma}$, determine $\widetilde{\mathcal{S}}_{\Gamma} w_{\Gamma} \in \widetilde{W}_{\Gamma}$, such that

$$
\left[\begin{array}{cccccc}
A_{I I}^{(1)} & A_{\Delta I}^{(1)^{T}} & & & & \widetilde{A}_{\Pi I}^{(1)^{T}} \\
A_{\Delta I}^{(1)} & A_{\Delta \Delta}^{(1)} & & & & \widetilde{A}_{\Pi \Delta}^{(1)^{T}} \\
& & \ddots & & & \vdots \\
& & & A_{I I}^{(N)} & A_{\Delta I}^{(N)^{T}} & \widetilde{A}_{\Pi I}^{(N)^{T}} \\
& & & A_{\Delta I}^{(N)} & A_{\Delta \Delta}^{(N)} & \widetilde{A}_{\Pi \Delta}^{(N)^{T}} \\
\widetilde{A}_{\Pi I}^{(1)} & \widetilde{A}_{\Pi \Delta}^{(1)} & \ldots & \widetilde{A}_{\Pi I}^{(N)} & \widetilde{A}_{\Pi \Delta}^{(N)} & \widetilde{A}_{\Pi \Pi}
\end{array}\right]\left[\begin{array}{c}
w_{I}^{(1)} \\
w_{\Delta}^{(1)} \\
\vdots \\
w_{I}^{(N)} \\
w_{\Delta}^{(N)} \\
w_{\Pi}
\end{array}\right]=\left[\begin{array}{c}
0 \\
\left(\widetilde{\mathcal{S}}_{\Gamma} w_{\Gamma}\right)_{\Delta}^{(1)} \\
\vdots \\
0 \\
\left(\widetilde{\mathcal{S}}_{\Gamma} w_{\Gamma}\right)_{\Delta}^{(N)} \\
\left(\widetilde{\mathcal{S}}_{\Gamma} w_{\Gamma}\right)_{\Pi}
\end{array}\right] .
$$

Here

$$
\widetilde{A}_{\Pi I}^{(i)}=R_{\Pi}^{(i)^{T}} A_{\Pi I}^{(i)}, \quad \widetilde{A}_{\Pi \Delta}^{(i)}=R_{\Pi}^{(i)^{T}} A_{\Pi \Delta}^{(i)}, \quad \forall i=1,2, \ldots, N,
$$

and

$$
\widetilde{A}_{\Pi \Pi}=\sum_{i=1}^{N} R_{\Pi}^{(i)^{T}} A_{\Pi \Pi}^{(i)} R_{\Pi}^{(i)} .
$$

Equation (19) can be viewed as the two-by-two block matrix of Equation (1), if we take the leading block-diagonal matrix with $N$ blocks, corresponding to the $N$ subdomains, to be the matrix $A$ of Equation (1), and the last diagonal block, corresponding to the coarse-level variables, to be the matrix $C$. Then the inverse of $\widetilde{\mathcal{S}}_{\Gamma}$ can be evaluated by Cholesky elimination as in Equation (3) of Section 2. We have

$$
\widetilde{\mathcal{S}}_{\Gamma}^{-1}=R_{\Gamma \Delta}^{T}\left(\sum_{i=1}^{N}\left[\begin{array}{ll}
0 & R_{\Delta}^{(i)^{T}}
\end{array}\right]\left[\begin{array}{cc}
A_{I I}^{(i)} & A_{\Delta I}^{(i)^{T}} \\
A_{\Delta I}^{(i)} & A_{\Delta \Delta}^{(i)}
\end{array}\right]^{-1}\left[\begin{array}{c}
0 \\
R_{\Delta}^{(i)}
\end{array}\right]\right) R_{\Gamma \Delta}+\Phi \mathcal{S}_{\Pi \Pi}^{-1} \Phi^{T},
$$


where the first part corresponds to $A^{-1}$ in Equation (3),

$$
\Phi=R_{\Gamma \Pi}^{T}-R_{\Gamma \Delta}^{T} \sum_{i=1}^{N}\left[\begin{array}{ll}
0 & R_{\Delta}^{(i)^{T}}
\end{array}\right]\left[\begin{array}{cc}
A_{I I}^{(i)} & A_{\Delta I}^{(i)^{T}} \\
A_{\Delta I}^{(i)} & A_{\Delta \Delta}^{(i)}
\end{array}\right]^{-1}\left[\begin{array}{c}
A_{\Pi I}^{(i)^{T}} \\
A_{\Pi \Delta}^{(i)^{T}}
\end{array}\right] R_{\Pi}^{(i)}
$$

corresponds to the matrix $\Phi$ in Equation (4), and $\mathcal{S}_{\Pi \Pi}$ is defined as in Equation (13), except for a case of many subdomains.

We define the operator $B_{\Gamma}$ by $B_{\Gamma}:=B_{\Delta} R_{\Gamma \Delta}$. From the fact that $R_{\Gamma \Delta} R_{\Gamma \Delta}^{T}=I$ and $R_{\Gamma \Pi} R_{\Gamma \Delta}^{T}=0$, we have,

$$
\begin{aligned}
B_{\Gamma} \widetilde{\mathcal{S}}_{\Gamma}^{-1} B_{\Gamma}^{T}= & \sum_{i=1}^{N}\left[\begin{array}{ll}
0 & B_{\Delta}^{(i)}
\end{array}\right]\left[\begin{array}{cc}
A_{I I}^{(i)} & A_{\Delta I}^{(i)^{T}} \\
A_{\Delta I}^{(i)} & A_{\Delta \Delta}^{(i)}
\end{array}\right]^{-1}\left[\begin{array}{c}
0 \\
B_{\Delta}^{(i)^{T}}
\end{array}\right] \\
& +\left(\sum_{i=1}^{N}\left[\begin{array}{ll}
0 & B_{\Delta}^{(i)}
\end{array}\right]\left[\begin{array}{cc}
A_{I I}^{(i)} & A_{\Delta I}^{(i)^{T}} \\
A_{\Delta I}^{(i)} & A_{\Delta \Delta}^{(i)}
\end{array}\right]^{-1}\left[\begin{array}{c}
A_{\Pi I}^{(i)^{T}} \\
A_{\Pi \Delta}^{(i)^{T}}
\end{array}\right] R_{\Pi}^{(i)}\right) \mathcal{S}_{\Pi \Pi}^{-1} \\
& \left(\sum_{i=1}^{N} R_{\Pi}^{(i)^{T}}\left[\begin{array}{ll}
A_{\Pi I}^{(i)} & A_{\Pi \Delta}^{(i)}
\end{array}\right]\left[\begin{array}{ll}
A_{I I}^{(i)} & A_{\Delta I}^{(i)^{T}} \\
A_{\Delta I}^{(i)} & A_{\Delta \Delta}^{(i)}
\end{array}\right]^{-1}\left[\begin{array}{c}
0 \\
B_{\Delta}^{(i)^{T}}
\end{array}\right]\right) \\
= & -\left(\widetilde{B}_{\Lambda \Lambda}-\widetilde{B}_{\left.\Lambda \Pi \mathcal{S}_{\Pi \Pi}^{-1} \widetilde{B}_{\Lambda \Pi}^{T}\right) .}\right.
\end{aligned}
$$

We can therefore write the FETI-DP system (18) as

$$
B_{\Gamma} \widetilde{\mathcal{S}}_{\Gamma}^{-1} B_{\Gamma}^{T} \lambda=-\left(d_{\Lambda}-\widetilde{B}_{\Lambda \Pi} \mathcal{S}_{\Pi \Pi}^{-1} g_{\Pi}\right) .
$$

The Dirichlet preconditioner used in the FETI-DP algorithms for solving Equation (22) is of the form $B_{D, \Gamma} \widetilde{\mathcal{S}}_{\Gamma} B_{D, \Gamma}^{T}$, where $B_{D, \Gamma}$ is defined by $B_{D, \Gamma}:=B_{D, \Delta} R_{\Gamma \Delta}$ and where $B_{D, \Delta}$ is constructed from subdomain operators $B_{D, \Delta}^{(i)}$ in the same way as $B_{\Delta}$. Each $B_{D, \Delta}^{(i)}$ is defined as follows: each row of $B_{\Delta}^{(i)}$ with a nonzero entry corresponds to a Lagrange multiplier connecting the subdomain $\Omega_{i}$ to a neighboring subdomain $\Omega_{j}$ at a point $x \in \Gamma_{i} \cap \Gamma_{j}$. Multiplying each such row of $B_{\Delta}^{(i)}$ with the positive scaling factor $\delta_{j}^{\dagger}(x)$ gives us $B_{D, \Delta}^{(i)}$. From the definition of $\widetilde{\mathcal{S}}_{\Gamma}$ in Equation (19), we can see that multiplying $\widetilde{\mathcal{S}}_{\Gamma}$ with a vector in range $\left(B_{D, \Gamma}^{T}\right)$ requires solving subdomain problems with Dirichlet boundary conditions. The preconditioned FETIDP operator with a Dirichlet preconditioner, is therefore of the form

$$
B_{D, \Gamma} \widetilde{\mathcal{S}}_{\Gamma} B_{D, \Gamma}^{T} B_{\Gamma} \widetilde{\mathcal{S}}_{\Gamma}^{-1} B_{\Gamma}^{T}
$$


We note that a different form of the FETI-DP operator,

$$
B_{D, \Delta} \mathcal{S}_{\Delta} B_{D, \Delta}^{T} B_{\Delta} \widetilde{\mathcal{S}}^{-1} B_{\Delta}^{T},
$$

was introduced in $[15,10]$, and used in the analysis of different FETI-DP algorithms. There $\mathcal{S}_{\Delta}$ is defined as the direct sum of subdomain Schur operators $\mathcal{S}_{\Delta}^{(i)}$ which are defined by: given $w_{\Delta}^{(i)} \in W_{\Delta}^{(i)}$, determine $\mathcal{S}_{\Delta}^{(i)} w_{\Delta}^{(i)} \in$ $W_{\Delta}^{(i)}$, such that

$$
\left[\begin{array}{cc}
A_{I I}^{(i)} & A_{\Delta I}^{(i)^{T}} \\
A_{\Delta I}^{(i)} & A_{\Delta \Delta}^{(i)}
\end{array}\right]\left[\begin{array}{c}
w_{I}^{(i)} \\
w_{\Delta}^{(i)}
\end{array}\right]=\left[\begin{array}{c}
0 \\
\mathcal{S}_{\Delta}^{(i)} w_{\Delta}^{(i)}
\end{array}\right]
$$

The operator $\widetilde{\mathcal{S}}$ in the FETI-DP operator (24) can be defined by Equation (19), except that we use vectors in the dual interface variable space $W_{\Delta}$, and set the coarse-level part of the right hand side of Equation (19) to zero.

From the definition of $\widetilde{\mathcal{S}}_{\Gamma}$ in Equation (19), we can see that $\mathcal{S}_{\Delta}$ and $\widetilde{\mathcal{S}}^{-1}$ are the restrictions of the operators $\widetilde{\mathcal{S}}_{\Gamma}$ and $\widetilde{\mathcal{S}}_{\Gamma}^{-1}$, respectively, to the space $W_{\Delta}$. Therefore, we have

$$
\mathcal{S}_{\Delta}=R_{\Gamma \Delta} \widetilde{\mathcal{S}}_{\Gamma} R_{\Gamma \Delta}^{T} ; \quad \widetilde{\mathcal{S}}^{-1}=R_{\Gamma \Delta} \widetilde{\mathcal{S}}_{\Gamma}^{-1} R_{\Gamma \Delta}^{T},
$$

which shows that the two preconditioned FETI-DP operators of Equations (23) and (24) are the same. The reason to introduce the form of FETI-DP operator given in Equation (23) is that it is more convenient in the study of the connection with the BDDC algorithms. Using the definition of $\mathcal{S}_{\Delta}^{(i)}$ in Equation (25), we find that $\widetilde{\mathcal{S}}_{\Gamma}^{-1}$, given in Equation (20), can be written as

$$
\widetilde{\mathcal{S}}_{\Gamma}^{-1}=R_{\Gamma \Delta}^{T} \mathcal{S}_{\Delta}^{-1} R_{\Gamma \Delta}+\Phi \mathcal{S}_{\Pi \Pi}^{-1} \Phi^{T},
$$

where $\mathcal{S}_{\Delta}^{-1}$ represents the direct sum of the $\mathcal{S}_{\Delta}^{(i)^{-1}}$.

\subsection{Neumann-Neumann Methods of the Same Flavor: BDDC}

In a Neumann-Neumann type algorithm, we solve the interface Schur complement problem: find $u_{\Gamma} \in \widehat{W}_{\Gamma}$, such that

$$
\widehat{\mathcal{S}} u_{\Gamma}=\sum_{i=1}^{N} R_{\Gamma}^{(i)^{T}} g_{\Gamma}^{(i)},
$$

with a preconditioner built from subdomain Neumann solvers, each possibly with a few constraints. In Equation $(27), g_{\Gamma}^{(i)}$ is the subdomain interface load 
vector, as in Equation (7), and $\widehat{\mathcal{S}}$ is the interface Schur complement operator, defined on the space $\widehat{W}_{\Gamma}$.

There are different ways of representing $\widehat{\mathcal{S}}$. Let us denote by $\mathcal{S}$ the Schur complemment operator defined on the space $W_{\Gamma}$, i.e., $\mathcal{S}$ is the direct sum of the $\mathcal{S}^{(i)}$, the subdomain Schur complements defined on the space $W_{\Gamma}^{(i)}$ as in Equation (6). Then $\widehat{\mathcal{S}}$, can be regarded as the restriction of $\mathcal{S}$ to the space $\widehat{W}$, and can therefore be written as

$$
\widehat{\mathcal{S}}=R_{\Gamma}^{T} \mathcal{S} R_{\Gamma}=\sum_{i=1}^{N}\left(R_{\Gamma}^{(i)^{T}} \mathcal{S}^{(i)} R_{\Gamma}^{(i)}\right) .
$$

$\widehat{\mathcal{S}}$ can also be written as the restriction of $\widetilde{\mathcal{S}}_{\Gamma}$ to the space $\widehat{W}$, and is therefore also of the form

$$
\widehat{\mathcal{S}}=\widetilde{R}_{\Gamma}^{T} \widetilde{\mathcal{S}}_{\Gamma} \widetilde{R}_{\Gamma}
$$

Remark 1 A one-level Neumann-Neumann preconditioner

$$
M^{-1}=R_{D, \Gamma}^{T} \mathcal{S}^{-1} R_{D, \Gamma}=\sum_{i=1}^{N} R_{D, \Gamma}^{(i)^{T}} \mathcal{S}^{(i)}{ }^{-1} R_{D, \Gamma}^{(i)},
$$

can be used for solving the interface problem (27), as in Section 3.1 for the two-subdomain case. We note that the use of the scaling means that we partition the residual on the interface and then, after solving the local problems, average the values obtained on the interface; cf. also [16, Section 1.3.4]. There are two problems with this approach. The $\mathcal{S}^{(i)}$ corresponding to interior subdomains typically are singular, introducing floating subdomains, and there is also no global component of the preconditioner. The first problem can be overcome by adding a small positive multiple of the mass matrices to the stiffness matrices of the subdomains when constructing the preconditioner. The lack of a global component, however, will make this preconditioner noncompetitive with a rate of convergence that will necessarily deteriorate with an increasing number of subdomains; see [16, Section 1.3.6]. Several successful two-level balancing Neumann-Neumann algorithm have been proposed to overcome this problem; see, e.g., [12] and [3]. Some of these algorithms have been used extensively for large scale problems.

The BDDC algorithm, first introduced by Dohrmann, [13, 2, 1], is a variant of the two-level Neumann-Neumann type preconditioner for solving the interface problem (27). In the BDDC preconditioner, the coarse-level problem is assembled from a special set of coarse basis functions, which are the 
minimum energy extension on the subdomains subject to sets of primal constraints; these coarse-level basis functions, in fact, correspond to the matrix $\Phi$ in the block Cholesky elimination in Equation (3). The primal constraints usually represent continuity at the subdomain corner and/or common edge or face averages across the interface, as in a FETI-DP algorithm.

Dohrmann's BDDC preconditioner is written in the form

$$
M_{B D D C}^{-1}=R_{D, \Gamma}^{T}\left(T_{0}+T_{s u b}\right) R_{D, \Gamma}
$$

see [14]. The coarse-level correction operator $T_{0}$ is defined by

$$
T_{0}=\Psi\left(\Psi^{T} \mathcal{S} \Psi\right)^{-1} \Psi^{T}
$$

where the coarse-level basis function matrix $\Psi$ is of the from

$$
\Psi=\left[\begin{array}{c}
\Psi^{(1)} \\
\vdots \\
\Psi^{(N)}
\end{array}\right]
$$

Each subdomain coarse-level basis function matrix $\Psi^{(i)}$ is determined by

$$
\left[\begin{array}{cc}
\mathcal{S}^{(i)} & C^{(i)^{T}} \\
C^{(i)} & 0
\end{array}\right]\left[\begin{array}{l}
\Psi^{(i)} \\
V^{(i)}
\end{array}\right]=\left[\begin{array}{c}
0 \\
R_{\Pi}^{(i)}
\end{array}\right],
$$

where $C^{(i)}$ represents the primal constraints of the subdomain $\Omega_{i}$ and each column of $V^{(i)}$ is a vector of Lagrange multipliers. The number of columns of each $\Psi^{(i)}$ is the same as the number of global coarse-level degrees of freedom. Only a few columns of each $\Psi^{(i)}$ are nonzero namely those supported on $\Omega_{i}$. To compute a nonzero column of $\Psi_{i}$, a subdomain Neumann problem is solved, which corresponds to a nonzero column of the matrix $R_{\Pi}^{(i)}$. We can also see from Equation (31), that each nonzero column of $\Psi^{(i)}$ is the minimum energy extension to the subdomain $\Omega_{i}$ setting one of the primal variables equal to 1 and all others equal to 0 .

The subdomain correction operator $T_{\text {sub }}$ is defined by

$$
T_{\text {sub }}=\sum_{i=1}^{N}\left[\begin{array}{ll}
R_{\Gamma}^{(i)^{T}} & 0
\end{array}\right]\left[\begin{array}{cc}
\mathcal{S}^{(i)} & C^{(i)^{T}} \\
C^{(i)} & 0
\end{array}\right]^{-1}\left[\begin{array}{c}
R_{\Gamma}^{(i)} \\
0
\end{array}\right],
$$

which gives subdomain corrections for which all the primal variables vanish. 
If we, as we always assume, have changed the variables, then the Lagrange multipliers are no longer needed to enforce the primal continuity constraints and the BDDC preconditioner (28) can be written as

$$
M_{B D D C}^{-1}=R_{D, \Gamma}^{T}\left\{R_{\Gamma \Delta}^{T} \mathcal{S}_{\Delta}^{-1} R_{\Gamma \Delta}+\Psi\left(\Psi^{T} \mathcal{S} \Psi\right)^{-1} \Psi^{T}\right\} R_{D, \Gamma},
$$

where we have replaced the subdomain correction operator $T_{\text {sub }}$, defined in Equation (32), by $R_{\Gamma \Delta}^{T} \mathcal{S}_{\Delta}^{-1} R_{\Gamma \Delta}$, since, after changing the variables, we can enforce zero primal parts simply by restricting the operators to the dual interface space $W_{\Delta}$. Similarly, Equation (31) takes the form:

$$
\left[\begin{array}{ccc}
A_{I I}^{(i)} & A_{\Delta I}^{(i)^{T}} & A_{\Pi I}^{(i)^{T}} \\
A_{\Delta I}^{(i)} & A_{\Delta \Delta}^{(i)} & A_{\Pi \Delta}^{(i) T^{T}} \\
A_{\Pi I}^{(i)} & A_{\Pi \Delta}^{(i)} & A_{\Pi \Pi}^{(i)}
\end{array}\right]\left[\begin{array}{l}
u_{I}^{(i)} \\
\Psi_{\Delta}^{(i)} \\
R_{\Pi}^{(i)}
\end{array}\right]=\left[\begin{array}{l}
0 \\
0 \\
\mathcal{S}_{\Pi \Pi}^{(i)} R_{\Pi}^{(i)}
\end{array}\right]
$$

where

$$
\Psi^{(i)}=\left[\begin{array}{c}
\Psi_{\Delta}^{(i)} \\
R_{\Pi}^{(i)}
\end{array}\right]=\left[-\left[\begin{array}{ll}
0 & I_{\Delta}^{(i)}
\end{array}\right]\left[\begin{array}{cc}
A_{I I}^{(i)} & A_{\Delta I}^{(i)}{ }^{T} \\
A_{\Delta I}^{(i)} & A_{\Delta \Delta}^{(i)}
\end{array}\right]^{-1}\left[\begin{array}{c}
A_{\Pi T^{T}}^{(i)^{T}} \\
A_{\Pi \Delta}^{(i)^{T}}
\end{array}\right] R_{\Pi}^{(i)}\right]
$$

and

$$
\mathcal{S}_{\Pi \Pi}^{(i)}=A_{\Pi \Pi}^{(i)}-\left[\begin{array}{ll}
A_{\Pi I}^{(i)} & A_{\Pi \Delta}^{(i)}
\end{array}\right]\left[\begin{array}{cc}
A_{I I}^{(i)} & A_{\Delta I}^{(i)^{T}} \\
A_{\Delta I}^{(i)} & A_{\Delta \Delta}^{(i)}
\end{array}\right]^{-1}\left[\begin{array}{c}
A_{\Pi I}^{(i)^{T}} \\
A_{\Pi \Delta}^{(i)^{T}}
\end{array}\right],
$$

which is a subdomain contribution to the operator $\mathcal{S}_{\Pi \Pi}$ of Equation (13). We can see, from Equations (34) and (35), that

$$
\Psi^{T} \mathcal{S} \Psi=\sum_{i=1}^{N} \Psi^{(i)^{T}} \mathcal{S}^{(i)} \Psi^{(i)}=\sum_{i=1}^{N} R_{\Pi}^{(i)^{T}} \mathcal{S}_{\Pi \Pi}^{(i)} R_{\Pi}^{(i)}=\mathcal{S}_{\Pi \Pi} .
$$

From the fact that $\Psi^{(i)}$ in Equation (35) is the same as the restriction of the matrix $\Phi$ of Equation (21) to the subdomain $\Omega_{i}$, we see that the matrix $\Psi$ in fact equals $\Phi$, except that $\Psi$ corresponds to interface vectors with distributed but continuous coarse-level variables and $\Phi$ corresponds to vectors with shared coarse-level degrees of freedom. From the assumption that $\sum_{j \in \mathcal{N}_{x}} \delta_{j}^{\dagger}(x)=1$ for every $x \in \Gamma$, we have $R_{D, \Gamma}^{T} \Psi=\widetilde{R}_{D, \Gamma}^{T} \Phi$. Therefore, from Equations (37) and (26), the BDDC preconditioner (33) can be written as,

$$
\begin{aligned}
M_{B D D C}^{-1} & =R_{D, \Gamma}^{T} R_{\Gamma \Delta}^{T} \mathcal{S}_{\Delta}^{-1} R_{\Gamma \Delta} R_{D, \Gamma}+R_{D, \Gamma}^{T} \Psi\left(\Psi^{T} \mathcal{S} \Psi\right)^{-1} \Psi^{T} R_{D, \Gamma} \\
& =\widetilde{R}_{D, \Gamma}^{T} R_{\Gamma \Delta}^{T} \mathcal{S}_{\Delta}^{-1} R_{\Gamma \Delta} \widetilde{R}_{D, \Gamma}+\widetilde{R}_{D, \Gamma}^{T} \Phi \mathcal{S}_{\Pi \Pi}^{-1} \Phi^{T} \widetilde{R}_{D, \Gamma}=\widetilde{R}_{D, \Gamma}^{T} \widetilde{\mathcal{S}}_{\Gamma}^{-1} \widetilde{R}_{D, \Gamma},
\end{aligned}
$$


and the preconditioned BDDC operator is of the form

$$
\widetilde{R}_{D, \Gamma}^{T} \widetilde{\mathcal{S}}_{\Gamma}^{-1} \widetilde{R}_{D, \Gamma} \widetilde{R}_{\Gamma}^{T} \widetilde{\mathcal{S}}_{\Gamma} \widetilde{R}_{\Gamma}
$$

\section{Matrix Analysis of FETI-DP and BDDC}

We first define jump and average operators on the space $\widetilde{W}_{\Gamma}$ by

$$
P_{D}:=B_{D, \Gamma}^{T} B_{\Gamma}, \quad E_{D}:=\widetilde{R}_{\Gamma} \widetilde{R}_{D, \Gamma}^{T} .
$$

They are complimentary projections:

Lemma $1 \quad E_{D}+P_{D}=I ; \quad E_{D}^{2}=E_{D}, P_{D}^{2}=P_{D} ; \quad E_{D} P_{D}=P_{D} E_{D}=0$.

Proof: Given a function $w_{\Gamma} \in \widetilde{W}_{\Gamma}$, we denote its coarse-level component by $w_{\Pi}$ and its dual subdomain components by $w_{\Delta}^{(i)}$, for $i=1,2, \ldots, N$. We have, from the definitions of $E_{D}$ and $\widetilde{R}_{D, \Gamma}$,

$$
E_{D} w_{\Gamma}=\widetilde{R}_{\Gamma} \widetilde{R}_{D, \Gamma}^{T} w_{\Gamma}=\widetilde{R}_{\Gamma}\left(R_{\Gamma \Pi}^{T} w_{\Pi}+\sum_{j=1}^{N} R_{\Gamma \Delta}^{T} R_{D, \Delta}^{(j)^{T}} w_{\Delta}^{(j)}\right)
$$

We see that the coarse-level component of $E_{D} w_{\Gamma}$ is still $w_{\Pi}$, and its dual subdomain components equal

$$
\left(E_{D} w_{\Gamma}\right)_{\Delta}^{(i)}(x)=\sum_{j \in \mathcal{N}_{x}} \delta_{j}^{\dagger}(x) w_{\Delta}^{(j)}(x), \quad \forall x \in \Gamma_{i} .
$$

From the definition of $P_{D}, B_{\Gamma}$, and $B_{D, \Gamma}$, we have

$$
P_{D} w_{\Gamma}=B_{D, \Gamma}^{T} B_{\Gamma} w_{\Gamma}=R_{\Gamma \Delta}^{T} B_{D, \Delta}^{T} B_{\Delta} R_{\Gamma \Delta} w_{\Gamma}=R_{\Gamma \Delta}^{T}\left(\sum_{j=1}^{N} B_{D, \Delta}^{(j)^{T}} B_{\Delta}^{(j)} w_{\Delta}^{(j)}\right) .
$$

We can see that the coarse-level component of $P_{D} w_{\Gamma}$ is zero, and that

$$
\left(P_{D} w_{\Gamma}\right)_{\Delta}^{(i)}(x)=\sum_{j \in \mathcal{N}_{x}} \delta_{j}^{\dagger}(x)\left(w_{\Delta}^{(i)}(x)-w_{\Delta}^{(j)}(x)\right), \quad \forall x \in \Gamma_{i} .
$$

By the assumption that $\sum_{j \in \mathcal{N}_{x}} \delta_{j}^{\dagger}(x)=1$, for any $x \in \Gamma$, we have

$$
\left(E_{D} w_{\Gamma}+P_{D} w_{\Gamma}\right)_{\Delta}^{(i)}(x)=\sum_{j \in \mathcal{N}_{x}} \delta_{j}^{\dagger}(x)\left(w_{\Delta}^{(j)}(x)+w_{\Delta}^{(i)}(x)-w_{\Delta}^{(j)}(x)\right)=w_{\Delta}^{(i)}(x) .
$$


We also note that the coarse-level component of $E_{D} w_{\Gamma}+P_{D} w_{\Gamma}$ equals $w_{\Pi}$. Therefore, for any vector $w_{\Gamma} \in \widetilde{W}_{\Gamma},\left(E_{D}+P_{D}\right) w_{\Gamma}=w_{\Gamma}$, i.e., $E_{D}+P_{D}=I$.

Equations (39) and (40) also show that range $\left(E_{D}\right) \subset \widehat{W}$ and that for any continuous function $w_{\Gamma} \in \widehat{W}_{\Gamma}, E_{D} w_{\Gamma}=w_{\Gamma}$. Therefore $E_{D}^{2}=E_{D}$. Since $E_{D}\left(E_{D}+P_{D}\right)=E_{D}$, we have $E_{D} P_{D}=0$. From Equation (42), we know that for any continuous interface function $w_{\Gamma}, P_{D} w_{\Gamma}=0$. Therefore $P_{D} E_{D}=0$, and since $P_{D}\left(E_{D}+P_{D}\right)=P_{D}$, we have $P_{D}^{2}=P_{D}$.

We note that the preconditioned FETI-DP operator (23) may become singular, when the matrix $B_{\Gamma}^{T}$ is not of full rank. This corresponds to the use of redundant Lagrange multipliers, see [8]. But we need not worry since the Lagrange multiplier $\lambda$ is always restricted to range $\left(B_{\Gamma}\right)$, which is orthogonal to the null space of $B_{\Gamma}^{T}$. For simplicity, we assume that a set of non-redundant Lagrange multipliers are used in the FETI-DP algorithm, i.e., that $B_{\Gamma}^{T}$ is of full rank. Therefore the preconditioned FETI-DP operator is always nonsingular. We note that Theorem 1, at the end of this section, applies equally well to the case of redundant Lagrange multipliers.

We now multiply the preconditioned FETI-DP operator (23) by $B_{\Gamma}^{T}$ on the left and remove the same factor on the right to obtain

$$
P_{D}^{T} \widetilde{\mathcal{S}}_{\Gamma} P_{D} \widetilde{\mathcal{S}}_{\Gamma}^{-1}
$$

It is easy to see that the two operators (23) and (43) have the same nonzero eigenvalues, since $B_{\Gamma}^{T}$ has full rank. We also multiply the preconditioned BDDC operator (38) by $\widetilde{R}_{\Gamma}$ on the left and remove the same factor on the right to obtain

$$
E_{D} \widetilde{\mathcal{S}}_{\Gamma}^{-1} E_{D}^{T} \widetilde{\mathcal{S}}_{\Gamma}
$$

which has the same nonzero eigenvalues as (38) since $\widetilde{R}_{\Gamma}$ has full rank.

We will now show that the two operators (43) and (44), and therefore the preconditioned FETI-DP and BDDC operators (23) and (38), have the same nonzero eigenvalues. Let $\varphi$ be an eigenvector of the operator (43) with the eigenvalue $\mu$, and let $\psi=E_{D} \widetilde{\mathcal{S}}_{\Gamma}^{-1} \varphi$. Then, from Lemma 1, we have,

$$
\begin{aligned}
E_{D} \widetilde{\mathcal{S}}_{\Gamma}^{-1} E_{D}^{T} \widetilde{\mathcal{S}}_{\Gamma} \psi & =E_{D} \widetilde{\mathcal{S}}_{\Gamma}^{-1} E_{D}^{T} \widetilde{\mathcal{S}}_{\Gamma} E_{D} \widetilde{\mathcal{S}}_{\Gamma}^{-1} \varphi=E_{D} \widetilde{\mathcal{S}}_{\Gamma}^{-1}\left(I-P_{D}^{T}\right) \widetilde{\mathcal{S}}_{\Gamma}\left(I-P_{D}\right) \widetilde{\mathcal{S}}_{\Gamma}^{-1} \varphi \\
& =E_{D} \widetilde{\mathcal{S}}_{\Gamma}^{-1} P_{D}^{T} \widetilde{\mathcal{S}}_{\Gamma} P_{D} \widetilde{\mathcal{S}}_{\Gamma}^{-1} \varphi-E_{D} P_{D} \widetilde{\mathcal{S}}_{\Gamma}^{-1} \varphi+E_{D} \widetilde{\mathcal{S}}_{\Gamma}^{-1}\left(I-P_{D}^{T}\right) \varphi
\end{aligned}
$$

where the first term on the right hand side equals $\mu \psi$, since $P_{D}^{T} \widetilde{\mathcal{S}}_{\Gamma} P_{D} \widetilde{\mathcal{S}}_{\Gamma}^{-1} \varphi=$ $\mu \varphi$. The second term vanishes since $E_{D} P_{D}=0$. In the third term, $\left(I-P_{D}^{T}\right) \varphi$ 
vanishes since $\varphi \in$ range $\left(P_{D}^{T}\right)$. We can therefore conclude that $\mu$ is also an eigenvalue of the operator (44) if $\psi \neq 0$.

We now examine the case when $\psi=E_{D} \widetilde{\mathcal{S}}_{\Gamma}^{-1} \varphi=0$. We find, since $E_{D}=I-P_{D}$, that $P_{D} \widetilde{\mathcal{S}}_{\Gamma}^{-1} \varphi=\widetilde{\mathcal{S}}_{\Gamma}^{-1} \varphi$. Therefore,

$$
P_{D}^{T} \widetilde{\mathcal{S}}_{\Gamma} P_{D} \widetilde{\mathcal{S}}_{\Gamma}^{-1} \varphi=P_{D}^{T} \varphi=\mu \varphi .
$$

Since $P_{D}^{T}$ is a projection, and $\varphi \in \operatorname{range}\left(P_{D}^{T}\right), P_{D}^{T} \varphi$ does not vanish and $\mu$ must equal 1.

Similarly, any eigenvalue, that differs from 1, of the operator (44) is also an eigenvalue of the operator (43); this is established in a similar way.

We can show that 1 is an eigenvalue of both operators. Since we know that 1 is a lower bound of the eigenvalues of the preconditioned FETI-DP operator $(23)$, see $[15,10]$, we know that 1 is also a lower bound of the nonzero eigenvalues of the operator (43). Since it is self-adjoint with respect to $<\cdot, \cdot>_{\widetilde{\mathcal{S}}_{\Gamma}^{-1}}$, we have

$$
1 \leq \min _{x} \frac{<x, P_{D}^{T} \widetilde{\mathcal{S}}_{\Gamma} P_{D} \widetilde{\mathcal{S}}_{\Gamma}^{-1} x>_{\widetilde{\mathcal{S}}_{\Gamma}^{-1}}}{<x, x>_{\widetilde{\mathcal{S}}_{\Gamma}^{-1}}}
$$

where we only consider $x$ for which the numerator does not vanish, since we only consider a bound of its nonzero eigenvalues. Since $P_{D}$ is a projection, 1 is one of its eigenvalues. Therefore 1 is also an eigenvalue of the operator $\widetilde{S}_{\Gamma}^{1 / 2} P_{D} \widetilde{S}_{\Gamma}^{-1 / 2}$, i.e., there is a nonzero vector $y$ such that $\widetilde{S}_{\Gamma}^{1 / 2} P_{D} \widetilde{S}_{\Gamma}^{-1 / 2} y=y$. Take $x=\widetilde{\mathcal{S}}_{\Gamma}^{1 / 2} y$ and we find that

$$
\begin{gathered}
\frac{<x, P_{D}^{T} \widetilde{\mathcal{S}}_{\Gamma} P_{D} \widetilde{\mathcal{S}}_{\Gamma}^{-1} x>_{\widetilde{\mathcal{S}}_{\Gamma}^{-1}}}{<x, x>\widetilde{\mathcal{S}}_{\Gamma}^{-1}}=\frac{<\widetilde{\mathcal{S}}_{\Gamma}^{1 / 2} y, P_{D}^{T} \widetilde{\mathcal{S}}_{\Gamma} P_{D} \widetilde{\mathcal{S}}_{\Gamma}^{-1} \widetilde{\mathcal{S}}_{\Gamma}^{1 / 2} y>_{\widetilde{\mathcal{S}}_{\Gamma}^{-1}}}{<\widetilde{\mathcal{S}}_{\Gamma}^{1 / 2} y, \widetilde{\mathcal{S}}_{\Gamma}^{1 / 2} y>_{\widetilde{\mathcal{S}}_{\Gamma}^{-1}}} \\
=\frac{<y, \widetilde{\mathcal{S}}_{\Gamma}^{-1 / 2} P_{D}^{T} \widetilde{\mathcal{S}}_{\Gamma} P_{D} \widetilde{\mathcal{S}}_{\Gamma}^{-1 / 2} y>}{<y, y>}=\frac{<\widetilde{\mathcal{S}}_{\Gamma}^{1 / 2} P_{D} \widetilde{\mathcal{S}}_{\Gamma}^{-1 / 2} y, \widetilde{\mathcal{S}}_{\Gamma}^{1 / 2} P_{D} \widetilde{\mathcal{S}}_{\Gamma}^{-1 / 2} y>}{<y, y>}=1,
\end{gathered}
$$

which means that equality has to hold in (45), and therefore 1 is the minimum nonzero eigenvalue of the operator (43). Similarly, we can also show that 1 is also the minimum eigenvalue of the operator (44). Thus, we have proved

Theorem 1 The preconditioned FETI-DP operator and the BDDC operator, given by Equations (23) and (38), respectively, have the same eigenvalues. 
Table 1: Condition number estimate $\kappa$, and iteration count iter., with changing number of subdomains, and different coarse-level basis functions, for $H / h=8$.

\begin{tabular}{|c||c|c||c|c||c|c|}
\hline \multicolumn{1}{|c||}{$\begin{array}{c}\text { Num. of subs } \\
n_{x} \times n_{y}\end{array}$} & \multicolumn{2}{c||}{ Corners + Edges } & \multicolumn{2}{c||}{ Edges only } & \multicolumn{2}{c|}{ Corners only } \\
\cline { 2 - 7 } & $\kappa$ & iter. & $\kappa$ & iter. & $\kappa$ & iter. \\
\hline $4 \times 4$ & $1.2(1.2)$ & $5(4)$ & $1.7(1.7)$ & $6(7)$ & $2.7(2.8)$ & $8(8)$ \\
$8 \times 8$ & $1.3(1.3)$ & $5(5)$ & $1.8(1.8)$ & $7(8)$ & $3.0(3.1)$ & $10(12)$ \\
$12 \times 12$ & $1.3(1.2)$ & $5(4)$ & $1.8(1.8)$ & $7(8)$ & $3.1(3.1)$ & $10(13)$ \\
$16 \times 16$ & $1.3(1.2)$ & $5(4)$ & $1.8(1.8)$ & $7(8)$ & $3.1(3.2)$ & $10(13)$ \\
$20 \times 20$ & $1.3(1.2)$ & $5(4)$ & $1.8(1.8)$ & $6(8)$ & $3.1(3.2)$ & $10(13)$ \\
\hline
\end{tabular}

Table 2: Condition number estimate and iteration count with changing $H / h$ and different coarse-level basis functions, for $4 \times 4$ subdomains.

\begin{tabular}{|c||c|c||c|c||c|c|}
\hline \multicolumn{1}{|c||}{} & \multicolumn{2}{c||}{ Corners + Edges } & \multicolumn{2}{c||}{ Edges only } & \multicolumn{2}{c|}{ Corners only } \\
\cline { 2 - 7 }$H / h$ & $\kappa$ & iter. & $\kappa$ & iter. & $\kappa$ & iter. \\
\hline 4 & $1.1(1.1)$ & $4(4)$ & $1.3(1.3)$ & $5(6)$ & $2.0(2.1)$ & $7(7)$ \\
8 & $1.2(1.2)$ & $5(4)$ & $1.7(1.7)$ & $6(7)$ & $2.7(2.8)$ & $8(8)$ \\
16 & $1.4(1.4)$ & $5(5)$ & $2.3(2.3)$ & $7(7)$ & $3.6(3.7)$ & $9(9)$ \\
32 & $1.7(1.7)$ & $6(6)$ & $3.0(3.1)$ & $8(8)$ & $4.6(4.7)$ & $10(10)$ \\
\hline
\end{tabular}

\section{$6 \quad$ Numerical Experiments}

Laplace's equation on a square domain with Dirichlet boundary conditions was solved with bilinear finite elements. A preconditioned conjugate gradient method was used to solve the interface problems derived from both BDDC and FETI-DP algorithms, and the iterations were stopped when the norm of the residual had been reduced by $10^{-6}$. Tables 1 and 2 show condition number estimates and iteration counts of the BDDC algorithm implemented as in (38), for varying numbers of subdomains and for different subdomain size. The corresponding results obtained from the implementation as in [13] are shown in parentheses. We see that both the condition number estimates and the iteration counts closely match for these two different implementation of BDDC. The scalability of the BDDC algorithm can also be observed.

In Tables 3 and 4, we compare the eigenvalue bounds of the BDDC 
Table 3: Eigenvalue bounds of the BDDC and the FETI-DP algorithms, with changing number of subdomains, and different coarse-level basis functions, for $H / h=8$.

\begin{tabular}{||c||cc|cc||cc|cc||}
\hline \multirow{2}{*}{\multicolumn{1}{|c||}{$\begin{array}{c}\text { Num. } \\
\text { of }\end{array}$}} & \multicolumn{3}{c||}{ Corners + Edges } & \multicolumn{4}{c||}{ Corners only } \\
\cline { 2 - 9 } subs. & \multicolumn{2}{|c|}{$\lambda_{\min }$} & \multicolumn{2}{c||}{$\lambda_{\max }$} & \multicolumn{3}{|c||}{$\lambda_{\min }$} & \multicolumn{2}{|c||}{$\lambda_{\max }$} \\
\cline { 2 - 9 } & BDDC & DP & BDDC & DP & BDDC & DP & BDDC & DP \\
$8 \times 8$ & 1.00 & 1.00 & 1.27 & 1.27 & 1.00 & 1.00 & 2.79 & 2.79 \\
$12 \times 12$ & 1.00 & 1.00 & 1.31 & 1.31 & 1.00 & 1.00 & 3.09 & 3.09 \\
$16 \times 16$ & 1.00 & 1.00 & 1.31 & 1.32 & 1.00 & 1.00 & 3.15 & 3.11 \\
$20 \times 20$ & 1.00 & 1.00 & 1.31 & 1.32 & 1.00 & 1.00 & 3.17 & 3.15 \\
\hline
\end{tabular}

Table 4: Eigenvalue bounds of BDDC and FETI-DP algorithms, with changing $H / h$, and different coarse-level basis functions, for $4 \times 4$ subdomains.

\begin{tabular}{|c||cc|cc||cc|cc||}
\hline \multirow{2}{*}{\multicolumn{1}{|c||}{$H / h$}} & \multicolumn{3}{c||}{ Corners + Edges } & \multicolumn{4}{c||}{ Corners only } \\
\cline { 2 - 9 } & \multicolumn{2}{|c|}{$\lambda_{\min }$} & \multicolumn{2}{c||}{$\lambda_{\max }$} & \multicolumn{2}{c||}{$\lambda_{\min }$} & \multicolumn{2}{c||}{$\lambda_{\max }$} \\
\cline { 2 - 9 } & BDDC & DP & BDDC & DP & BDDC & DP & BDDC & DP \\
\hline 4 & 1.00 & 1.00 & 1.11 & 1.11 & 1.00 & 1.00 & 2.07 & 2.07 \\
8 & 1.00 & 1.00 & 1.27 & 1.27 & 1.00 & 1.00 & 2.79 & 2.79 \\
16 & 1.00 & 1.00 & 1.48 & 1.48 & 1.00 & 1.00 & 3.64 & 3.64 \\
32 & 1.00 & 1.00 & 1.73 & 1.73 & 1.00 & 1.00 & 4.64 & 4.64 \\
\hline
\end{tabular}

algorithm with those of the FETI-DP algorithm, for the operators given in Equations (38) and (23), respectively. We note that in the BDDC and FETI-DP implementations, we have changed to a basis which includes the edge averages. We see that the lower and upper eigenvalue bounds of the two algorithms match very well, for different meshes. In all experiments, the lower and upper eigenvalue bounds are estimated by using the tridiagonal Lanczos matrix generated by the preconditioned conjugate gradient method. To simplify the implementation, we have included the corner nodes in the primal set for both these BDDC and FETI-DP implementations. 


\section{References}

[1] Clark R. Dohrmann. A preconditioner for substructuring based on constrained energy minimization. SIAM J. Sci Comput., 25(1):246$258,2003$.

[2] Clark R. Dohrmann. A study of domain decomposition preconditioners. Technical Report SAND2003-4391, Sandia National Laboratories, Albuquerque, New Mexico, 2003.

[3] Maksymilian Dryja and Olof B. Widlund. Schwarz methods of Neumann-Neumann type for three-dimensional elliptic finite element problems. Comm. Pure Appl. Math., 48(2):121-155, February 1995.

[4] Charbel Farhat, Michel Lesoinne, Patrick Le Tallec, Kendall Pierson, and Daniel Rixen. FETI-DP: A dual-primal unified FETI method part I: A faster alternative to the two-level FETI method. Internat. J. Numer. Methods Engrg., 50:1523-1544, 2001.

[5] Charbel Farhat, Michel Lesoinne, and Kendall Pierson. A scalable dualprimal domain decomposition method. Numer. Linear Algebra Appl., $7(7-8): 687-714,2000$.

[6] Yannis Fragakis and Manolis Papadrakakis. The mosaic of high performance domain decomposition methods for structural mechanics: Formulation, interrelation and numerical efficiency of primal and dual methods. Comput. Methods Appl. Mech. Engrg, 192(35-36):3799-3830, 2003.

[7] Axel Klawonn and Oliver Rheinbach. A parallel implementation of Dual-Primal FETI methods for three dimensional linear elasticity. Technical report, University of Essen, 2004. In preparation.

[8] Axel Klawonn and Olof B. Widlund. FETI and Neumann-Neumann iterative substructuring methods: Connections and new results. Comm. Pure Appl. Math, 54:57-90, January 2001.

[9] Axel Klawonn and Olof B. Widlund. Dual-Primal FETI methods for linear elasticity. Technical Report TR2004-855, Department of Computer Science, Courant Institute, September 2004.

[10] Axel Klawonn, Olof B. Widlund, and Maksymilian Dryja. Dual-primal FETI methods for three-dimensional elliptic problems with heterogeneous coefficients. SIAM J. Numer. Anal., 40(1):159-179, April 2002. 
[11] Axel Klawonn, Olof B. Widlund, and Maksymilian Dryja. Dual-Primal FETI methods with face constraints. In Luca Pavarino and Andrea Toselli, editors, Recent Developments in Domain Decomposition Methods, Lecture Notes in Computational Science and Engineering, Volume 23, pages 27-40. Springer, 2002.

[12] Jan Mandel. Balancing domain decomposition. Comm. Numer. Meth. Engrg., 9:233-241, 1993.

[13] Jan Mandel and Clark R. Dohrmann. Convergence of a balancing domain decomposition by constraints and energy minimization. Numer. Linear Algebra Appl., 10(7):639-659, 2003.

[14] Jan Mandel, Clark R. Dohrmann, and Radek Tezaur. An algebraic theory for primal and dual substructuring methods by constraints. Appl. Numer. Math.

[15] Jan Mandel and Radek Tezaur. On the convergence of a dual-primal substructuring method. Numer. Math., 88(3):543-558, January 2001.

[16] Andrea Toselli and Olof B. Widlund. Domain Decomposition Methods Algorithms and Theory, volume 34 of Springer Series in Computational Mathematics. Springer Verlag, Berlin-Heidelberg-New York, 2004.

[17] Xuemin Tu. Three-level BDDC in two dimensions. Technical Report TR2004-856, Department of Computer Science, Courant Institute, November 2004. 